\title{
Nano-CT and Electron Microscopy Cross-correlative Study of Tritiated LiAlO2 Pellet Nanopores
}

Bethany Matthews, Alan Albrecht, Timothy Pope and David Senor

Pacific Northwest National Laboratory, Richland, Washington, United States

Tritium producing burnable absorber rods (TPBAR) are used for production of the hydrogen isotope tritium $\left({ }^{3} \mathrm{H}\right)$. These rods are formed by concentric cylindrical layers; the main tritium producing part being the $\mathrm{LiAlO}_{2}$ pellet layer in which ${ }^{6} \mathrm{Li}$, under neutron irradiation, is converted into ${ }^{3} \mathrm{H}$ and ${ }^{4} \mathrm{He}$ ions. Pellets after irradiation become highly porous (Figure1b and 1c), with pores on the scale of 1um or smaller. Such pores can significantly affect the performance of a material, so it is important to characterize the morphology and networking of these pores and grain boundaries and to understand how irradiation may alter the chemical uniformity spatially in 3D. X-ray Computed Tomography (CT) has been an important technique for non-destructive analysis of the physical 3D structure; however, features in this technique are distinguished mainly by differences in density because it does not collect any chemical information.

Previous work shows nano-CT (nCT) is capable of successfully mapping microstructural features down to $\sim 100 \mathrm{~nm}$ in this material.[1] The work also noted that regions around many pores appeared denser than the general matrix, especially around the larger pores $(\sim 1 \mathrm{um})$, most of which exist prior to irradiation. In order to determine whether these regions are artifacts from the CT measurements or elemental variation, it is necessary to conduct chemical analyses. Here we examine a correlative microscopy approach to map 3D elemental information onto 3D microstructure information obtained by $\mathrm{nCT}$ and focused ion beam (FIB) serial sectioning with scanning electron microscopy (SEM) imaging. Both unirradiated and irradiated samples are examined to determine differences that might have arisen from irradiation.

A Helios 660 dual beam Ga FIB-SEM is used to extract and shape nCT needles. To obtain the best resolution and highest quality data in the lab-based Zeiss Xradia Ultra $810 \mathrm{nCT}$ [2], small volumes of the pellet material are extracted from site-specific regions and shaped into cylinders as described in reference [1]. After the nCT measurement and reconstruction, the FIB-SEM is used to image and to obtain energy dispersive $\mathrm{x}$-ray spectroscopy analysis of the volume during serial sectioning. As Li is difficult to detect in most EDS detectors, time of flight secondary ion mass spectroscopy is employed for examining the distribution of $\mathrm{Li}$. 


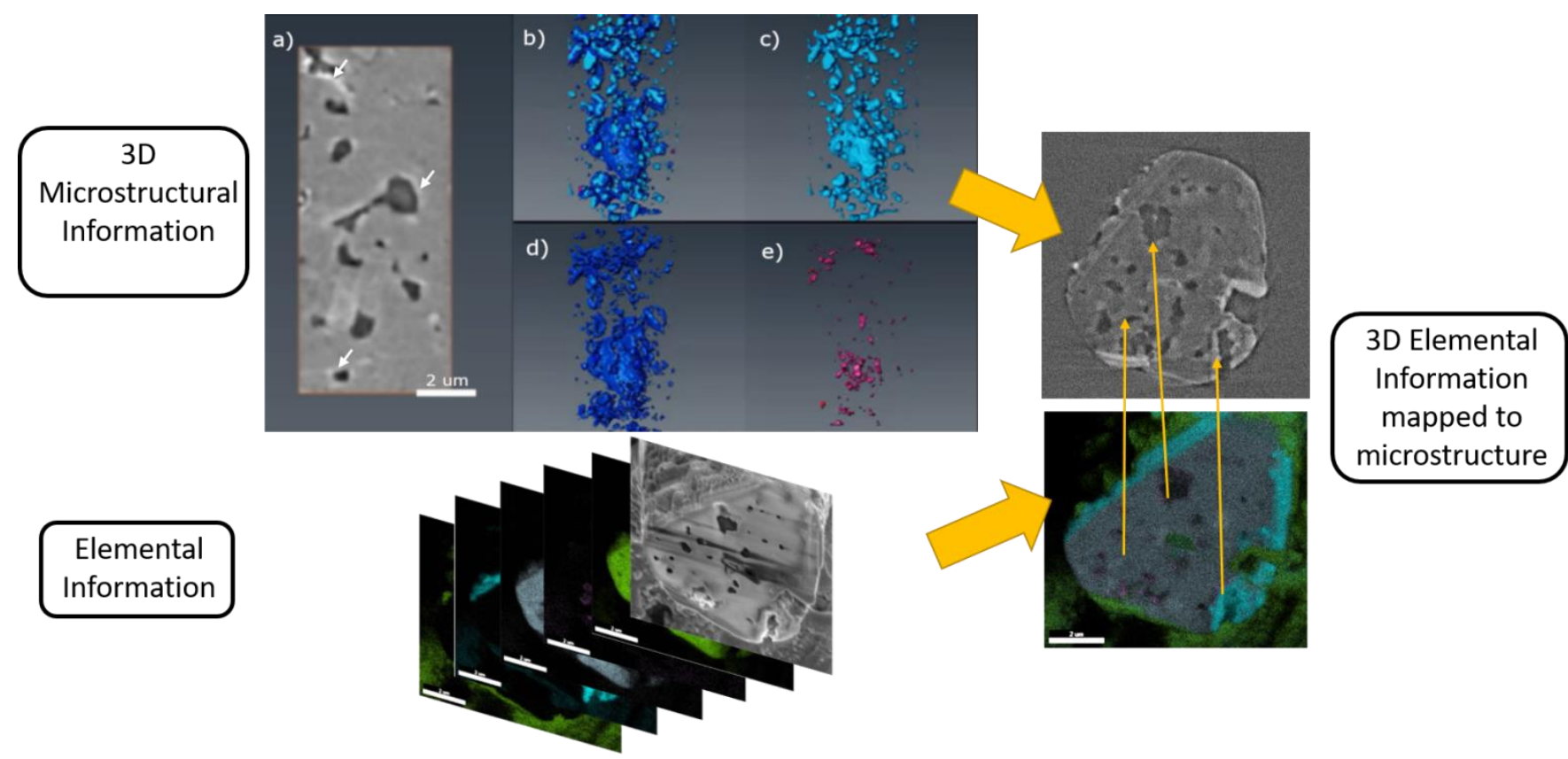

Figure 1. A schematic overview of the work combining microstructural and elemental data. Microstructural data includes a) orthogonal slice of reconstructed $\mathrm{nCT}$ data with segmented volumes c) pores, d) regions slightly denser than the general matrix (also indicated by the white arrows in a)), e) highly dense particles, and $b$ ) the combine segments. Elemental information in the form of EDS maps obtained between serial cuts with a FIB. These two types of information can be combined to inform about features previously observed in pellet samples.

\section{References}

[1] Matthews, B. E. et al. 2020. "Nano-Computed Tomography for Microstructural Characterization of TPBAR Components." Technical report PNNL-30558.

[2] https://www.zeiss.com/microscopy/us/products/x-ray-microscopy/xradia-810-ultra.html

[3] We would like to thank Marc Weber at Washington State University for his expertise and use of the Zeiss Xradia Ultra 810. 